\title{
My Wish for a Global Research Agenda in Nursing
}

\author{
Afaf Ibrahim Meleis
}

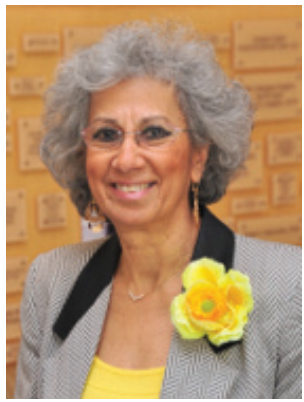

I have a wish! I wish for a strong voice for nurses in influencing health care globally. Since such a voice must be fortified by evidence, then my wish is that nursing research should be driven by a focused and coherent research agenda that will make a difference to the wellbeing of all populations, particularly the vulnerable and marginalized. To be able to make this wish come true, we need to identify health care priorities, develop mechanisms for collaboration, utilize strategies to integrate findings and find resources to fund these efforts. But let me first address the rationale for a focused research agenda. Let us look at the health care trends that appear to be shared around the world.

World populations are growing older due to the many scientific discoveries that have extended life expectancy. However, with advanced age, health care needs are changing and new challenges are appearing/arising. Therefore, there is an urgent need to partner with communities and to provide a continuum of care that is mostly based outside hospitals and in communities. There is also an increased demand for long-term care as well as home care. These trends inspire many research questions.

Here is another definite trend. Through external immigration or internal migration, more and more people are moving into urban areas. It is estimated that by the year 2050 at least $66 \%$ of the world population will be urbanized(1). Increasing urbanization provides better options for education and work opportunities. Nonetheless, accompanying these opportunities are some fundamental urban issues such as poverty, inaccessible health care and environmental hazards including pollution and accidents. Limited lighting and inaccessible transportation lead to unsafe environments and sedentary lifestyles, which in turn attract chronic diseases such as heart disease, asthma and diabetes. Moreover, healthy nutrition choices become more limited and are substituted with processed food and less fresh fruit and vegetables. There is also increased violence in urban areas as well as compromised human connectedness leading to mental health issues.

These trends could and should shape national research agendas for nursing scholars. There are some positive trends that could further support the development of a shared set of research goals. Global collaboration is facilitated by easier modes of transportation and more advanced communication infrastructures. Within this context of shared population trends and potential for global collaboration, we could develop a global agenda for nursing research which focusses on vulnerable populations, to support the frail elderly populations as well as promote health for those elderly who are healthy. This goal could be directed at enhancing their sense of wellbeing, preventing illness and decreasing their disparities. It also could include a focus on developing culturally competent care aimed at decreasing vulnerability and developing, implementing and evaluating the best practices for preventing illness and promoting health and wellbeing. And finally, our global agenda could focus on defining and promoting a culture of health that empowers all populations to make healthy choices in their day to day lives that lead to long term wellbeing ${ }^{(2)}$.

To make an impact, to change policies and to make a difference require articulating coherent frameworks to guide the development of research agendas that reflect global health priorities as well as local priorities. In other words, theory should drive questions and findings should modify and extend theories. Research programs that affect policies for population health and health care should be interdisciplinary and interprofessional. The goals for advancing knowledge should be guided by a framework with a set of principles that promote equity and justice.

My wish is to see that research projects developed around the globe are informed by an agenda that purports to uncover the responses and experiences of the population to health and wellness issues. It is also to answer coherently 
these relevant health care questions related to major population trends. More specifically, these global research programs should reflect the emerging trends of globalization, urbanization, aging, noncommunicable diseases as well as increasing technological innovations. The ultimate goal is to develop evidence based best practices that ensure access to quality health care for all populations, particularly the vulnerable and marginalized. Coherent and theory driven programs of research based on global trends and local health care priorities resulting in well supported findings, could make a difference in giving nursing a strong voice in influencing global health care agenda and outcomes.

\section{References}

1. Department of Economic and Social Affairs of the United Nations Secretariat. World Urbanization Prospects Report. New York: United Nations; 2014.

2. Lavizzo-Mourey R. Building a Culture of Health: 2014. Princeton (NJ): Robert Wood Johnson Foundation; 2014.

Afaf Ibrahim Meleis is a Professor of Nursing and Sociology at the University of Pennsylvania School of Nursing, Philadelphia, PA, United States, former Dean of Nursing and Former Director of the School's WHO Collaborating Center for Nursing and Midwifery Leadership. E-mail: meleis@nursing.upenn.edu 